\title{
CONTROL OF BOUNDARY LAYER BUILD-UP IN AN IMPELLER
}

\section{NITHIYAPATHI \& B. AKILAN}

Karpagam Academy of Higher Education, Coimbatore, Tamil Nadu, India

\begin{abstract}
The operation of the power producing engine is directly proportional to smooth and efficient compressor operation. The efficiency of an engine is directly related to the compression ratio of the compressor and expansion ratio of the turbine. So the need of understanding pressure loss due to low performance and poor reliability, slipping of flow is essential in today scenario. An investigation of flow over a centrifugal impeller is aimed to control the boundary layer build-up due to side slip of working fluid. For the purpose of flow analysis a CAD model of an impeller was designed using CATIA V5 and structural analysis has carried out for the designed model to check the maximum stress. As the main objective is being fluid flow analysis, the flow volume for the impeller model was generated using GAMBIT software and analysis was carried out using FLUENT.
\end{abstract}

KEYWORDS: Centrifugal Compressor, Impeller Design, Slots \& Boundary Layer

Received: Jan 18, 2019; Accepted: Feb 08, 2019; Published: Mar 13, 2019; Paper Id.: IJMPERDAPR201950

\section{INTRODUCTION}

Centrifugal compressors have its extensive usage over the last century and in rotorcraft engines over the last 68 years for energy production. The designs of compressors are highly experimental, employ scaling strategies and rely on prototype testing to reach design goals. This design process reverberate the facts that the flow in these machines is highly complex [2]. The primary objective of the compressor is to compress the working fluid either it is gas or vapor for the purpose of increasing the pressure of any compressible fluid. The compression of the fluid is done by reduction of fluid specific volume while the fluid is allowed to pass through the compressor. So that it delivers a higher pressure level of fluid than its original pressure for power generation [1].

Dheeraj Sagar et.al, investigated turbulent separated flow in an axisymmetric diffuser due to skin friction. They mainly concentrated in flow separation as well as to downstream reattachment. They found and concluded that the pressure distribution becomes uniform and separation was getting delayed as diffuser half-angle $\alpha$ decrease [3]. Yangwei Liu et.al, performed numerical studies for both design and off-design flow conditions. They inquired the effects of boundary layer suction on corner separation in a highly loaded compressor cascade. They proposed two new BLS slot configurations and a total of five suction slot configurations. Using this compound slot configuration, the maximum reduction of total pressure loss was obtained at 7 degree incidence could be $39.4 \%$ [4]. Yu-Tai Lee, et.al. predicted the flow separations at the shroud in front of the leading edges ofthe blade. An improvement in impeller performance would require reducing this shroud flow separation. They experience the difficulty due to the boundary layer at large curvature of the shroud as it approached the blade might be partially responsible for the flow separation [5]. Several investigations and studies on flow separation was made earlier at the diffuser and impeller shroud, and of course at impeller blade. Generally velocity variations from hub to shroud resulting changes in flow directions complicate the design procedure for centrifugal compressors. C.H.Wu [2] has 
presented the three dimensional theory in an impeller. It is composed of two solutions, one in the meridional surface (hub-to-shroud), and the other in the stream surface of revolution (blade-to-blade). Based on his theory flow analysis was carried to find the velocity drop point to encounter the loss in kinetic energy at impeller.

The present study concentrated on investigating the pressure losses due to incidence loss that is the direction of the relative velocity of fluid at inlet does not match with the inlet blade angle and therefore fluid cannot enter the blade passage smoothly by gliding along the blade surface. It causes a sudden discontinuity in fluid properties and flow parameters resulting escaping from the flow path. To carry over the study, an investigation was done for a compressor shape for $120 \mathrm{KW}$ micro-turbine output.

\section{COMPUTATIONAL METHODOLOGY}

A single shaft micro gas turbine is considered here for the generator utilization which has a single shaft that appended to the compressor and turbine. The work of the shaft is stuck to be same, however the work done has been utilized and it is a different amount of transmitted power along the joint segments of the shaft. The part of the power generated by gas turbine is consumed by the compressor in its work is assumed to 55 to 60 percentages [7]. For the greater safe side the consumption of power is taken as $60 \%$ at that point the power created is calculated as $120 \mathrm{~kW}$ which is an outstanding force of $40 \%$ from the shaft control delivered by a gas turbine. Hence the shaft power to operate the compressor would be $180 \mathrm{~kW}$.

For the finest rotational speed the microturbine typically operates at $45 \mathrm{krpm}$ to $100 \mathrm{krpm}$ because the maximum airflow will not exceed Mach number of 1 . The diameter of the shaft from the below formula it is found that $d_{s}=0.0152 \mathrm{~m}$. for safety consideration it was assumed to be $20 \mathrm{~mm}(0.02 \mathrm{~m})$. The impeller hub, which is attached to the shaft is having a length of $42 \mathrm{~mm}$ (mathematically calculated). Here the material is considered to be the usual one, that is $42 \mathrm{CrMo} 4 \mathrm{~V}$.

$$
d_{s}^{3}=\frac{16}{\pi \times \tau_{\max }} \sqrt{\left(K_{b} \times M_{b}\right)^{2}+\left(K_{t} \times M_{t}\right)^{2}}
$$

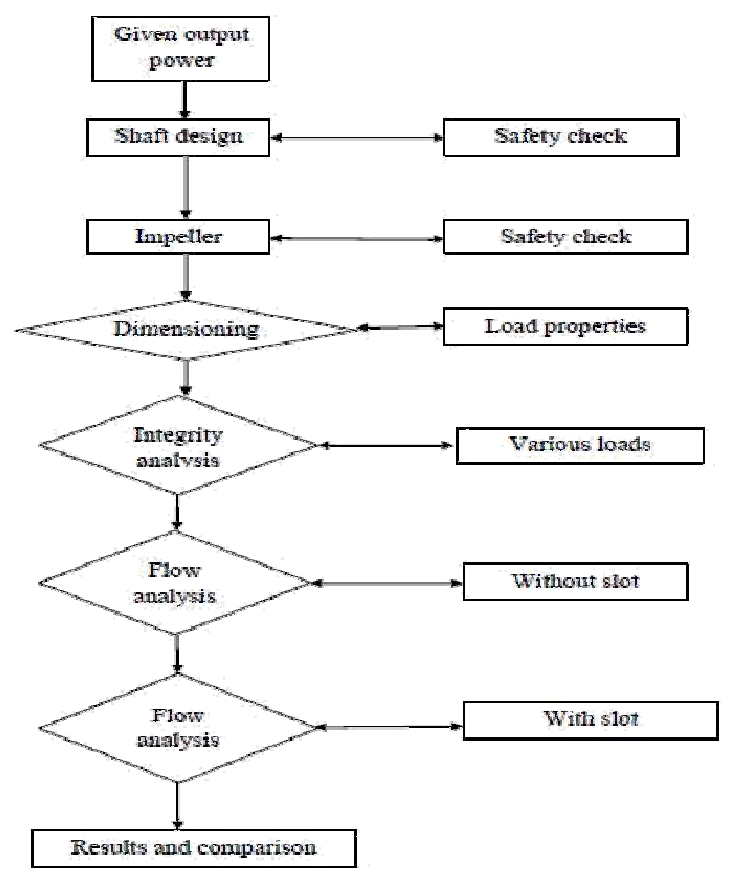

Figure 1: 2D Sketch of Shaft Design 


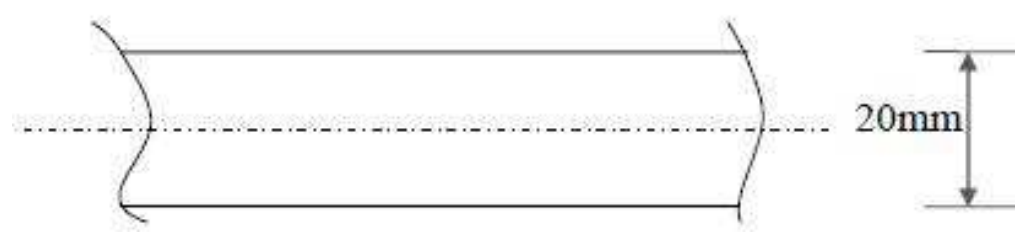

Figure 2: Computational Methodology

Limiting yield stress had checked for safety with respect to torsion applied. It was known and found that torque given is not exceeding the limiting torque yield stress value. For finding out impeller outside and inside diameter the inlet and outlet temperature of the impeller was first found, outside tip velocity of the impeller evaluated using air inlet temperature (equation 2). The calculated values were tabulated below.

$$
T_{2}-T_{1}=\varphi \mu \frac{v_{2}^{2}}{c_{p}}
$$

Table 1: Computed Results for Impeller Design

\begin{tabular}{|c|c|}
\hline \multicolumn{2}{|c|}{ Implicit Values } \\
\hline Parameters & Values \\
\hline Microturbine Output & $120 \mathrm{~kW}$ \\
\hline $\begin{array}{l}\text { Optimal rotational speed of } \\
\text { Microturbine }\end{array}$ & $45 \mathrm{krpm}$ to $100 \mathrm{krpm}$ \\
\hline $\begin{array}{l}\text { 42CrMo4V Ultimate Tensile } \\
\text { Strength }\end{array}$ & $1100 \mathrm{MPa}$ \\
\hline Air Inlet temperature & $298 \mathrm{~K}$ \\
\hline
\end{tabular}

\begin{tabular}{|c|c|}
\hline \multicolumn{2}{|c|}{ Calculated Values } \\
\hline Parameters & Values \\
\hline Torsional Moment & $38.1978 \mathrm{Nm}$ \\
\hline $\begin{array}{l}\text { Impeller to Shaft applied Torsional } \\
\text { stress }\end{array}$ & $\begin{array}{c}452278.86 \\
\mathrm{~N} / \mathrm{m}^{2}\end{array}$ \\
\hline Applied Torque & $71.039 \mathrm{Nm}$ \\
\hline Air Outlet Temperature & $488.88 \mathrm{~K}$ \\
\hline
\end{tabular}

\section{DESIGN AND STRUCTURAL ANALYSIS}

The design specifications were taken from the calculated values that were obtained using mathematical formulae. As per the targeted power produce the required shaft diameter is $20 \mathrm{~mm}$ and outer diameter of the impeller was about $181 \mathrm{~mm}$. Further design of the compressor impeller is developed using a solid multi - section to fix the blades in the compressor disk. For the designated values the impeller thickness to the shaft was $42 \mathrm{~mm}$ and outflow thickness was $6.94 \mathrm{~mm}$ and the blade angle was 23.35 degrees. Number of blades on the impeller would find by slip factor and air intake velocity. The design parameters were tabulated below.

Table 2: Compressor Geometry Parameter

\begin{tabular}{|l|c|c|}
\hline \multicolumn{1}{|c|}{ Objects } & Parameter & Magnitude \\
\hline Shaft diameter & $\mathrm{ds}(\mathrm{mm})$ & 20 \\
\hline Impeller inner diameter & $\mathrm{d} 1(\mathrm{~mm})$ & 73.3 \\
\hline Impeller outer diameter & $\mathrm{d} 2(\mathrm{~mm})$ & 181 \\
\hline Impeller thickness to shaft & $\mathrm{h}(\mathrm{mm})$ & 42 \\
\hline Impeller outflow thickness & $\mathrm{b}(\mathrm{mm})$ & 6.94 \\
\hline Blade/vane degree & $\beta($ degree $)$ & 23.35 \\
\hline Number of blades/vanes & $\mathrm{n}$ & 11 \\
\hline
\end{tabular}

Using the evaluated geometrical parameter, a three dimensional model have been drawn as shown in figure below. Figure 3 gives 3D view of designed impeller.

Structural analysis for designated centrifugal impeller is carried out here by using ANSYS software. This analysis is also said to be as Finite Element Analysis which is a mathematical portrayal of a physical framework comprising a part/ assembly (model), material properties and applicable boundary conditions (collectively referred to as pre-processing), the 
solution of that mathematical depiction (solving), and the study of the results of that solution (post-processing).

For pre-processing FEA software typically uses a CAD representation of the physical model and breaks it down into small pieces called finite "elements". This process is called - meshing\|. Before meshing, at first, the properties of the element should be defined properly which includes young's modulus, poisson's ratio, and density for the structural analysis.

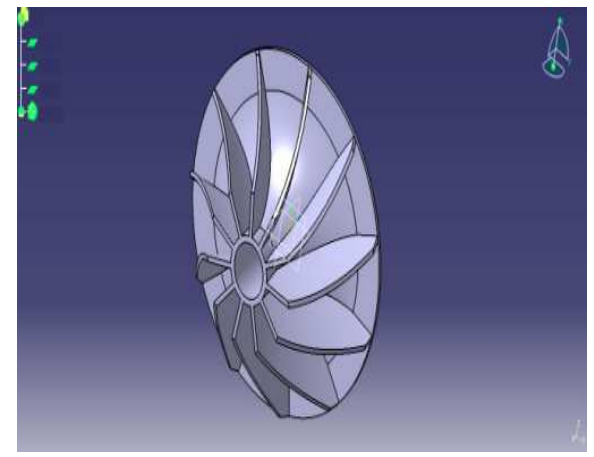

Figure 3: 3D Model of an Impeller

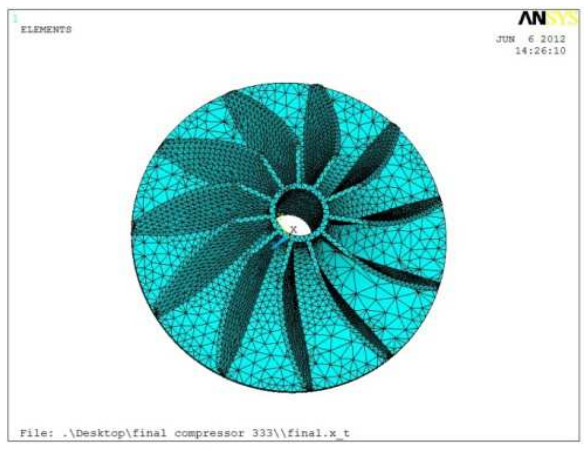

Figure 4: Impeller Mesh

More attention was focused on mesh specification and mesh suitability (refer figure 4) i.e. appropriate choice of element types and mesh density to give a solution to the required degree of accuracy. The material for the impeller (blade) was chosen into two different materials (commonly used for small size compressor) for the analysis [10] was tabulated below.

Table 3: Material Properties for Impeller

\begin{tabular}{|l|c|c|c|c|}
\hline \multicolumn{1}{|c|}{ Material } & $\begin{array}{c}\text { Young's } \\
\text { Modulus, E }(\mathbf{P a})\end{array}$ & $\begin{array}{c}\text { Poisson's } \\
\text { ratio, } \boldsymbol{\gamma}\end{array}$ & Density, $\boldsymbol{\rho}\left(\mathbf{K g} / \mathbf{m}^{\mathbf{3}}\right)$ & $\begin{array}{c}\text { Ultimate Tensile } \\
\text { Strength, UTS MPa }\end{array}$ \\
\hline Titanium AlloyTi-b-120UCA & $0.102 \mathrm{E} 12$ & 0.3 & 4850 & 1147 \\
\hline Stainless Steel Alloy-304 & $0.193 \mathrm{E} 12$ & 0.29 & 8030 & 1675 \\
\hline
\end{tabular}

It was noted that the Loads, constraints and Deformations were experienced in the compressor mainly due to two kind forces (refer figure 5). One was its inertial loads caused by own material property due to the movement of rotation. These parameters are dependent to properties such as mass and density of a material and speed of rotational objects. The region was on all parts of the body. In the meantime the issues, including three dimensional axisymmetric solids of transformation were subjected to Axisymmetric loading. Revolving bodies like compressor can be dissected by introducing centrifugal forces in the body force term.

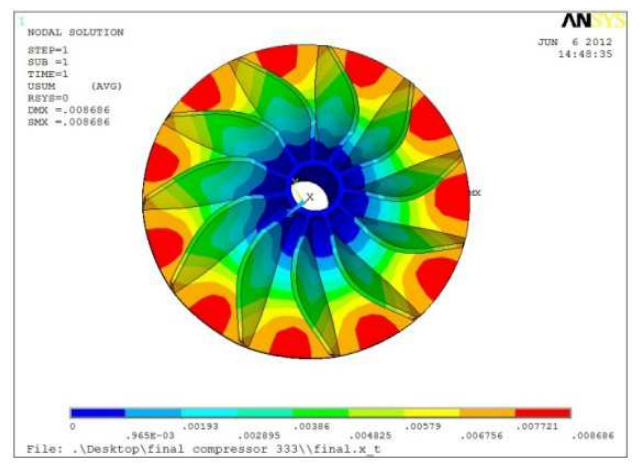

Figure 5: Deformation Due to Blade Speed

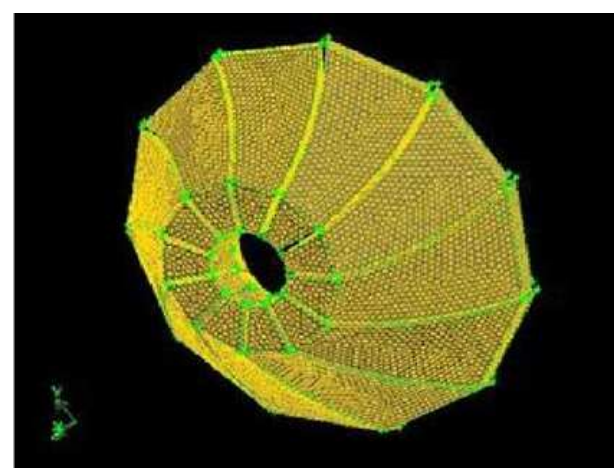

Figure 6: Flow Path-Meshed Volume with Slot 


\section{FLOW ANALYSIS}

Analysis begins with a mathematical model of a physical problem using CFD. It deals with conservation of matter, momentum, and energy that must be satisfied throughout the region of interest. Fluid properties are modelled empirically and Streamlining suppositions are made with a specific end goal to make the issue tractable. Also, it provides appropriate initial and boundary conditions for the problem. The analysis region is modelled using GAMBIT for the designated compressor stage where the fluid flow carries over. Normally the flow enters at inlet and exits radially at the tip, so the required boundary conditions at the inlet and outlet is set to get output as the pressure parameter.

The complete mesh of 3D hexahedral was done and the boundary conditions were given according to the flow conditions.
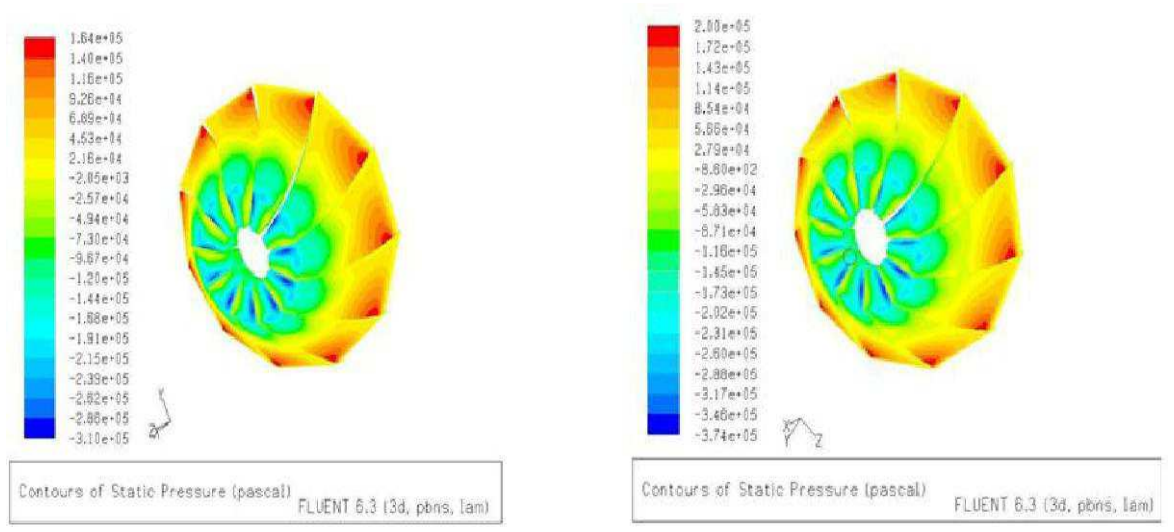

Figure 7: Static Pressure of Impeller Without Slot Vs With Slot
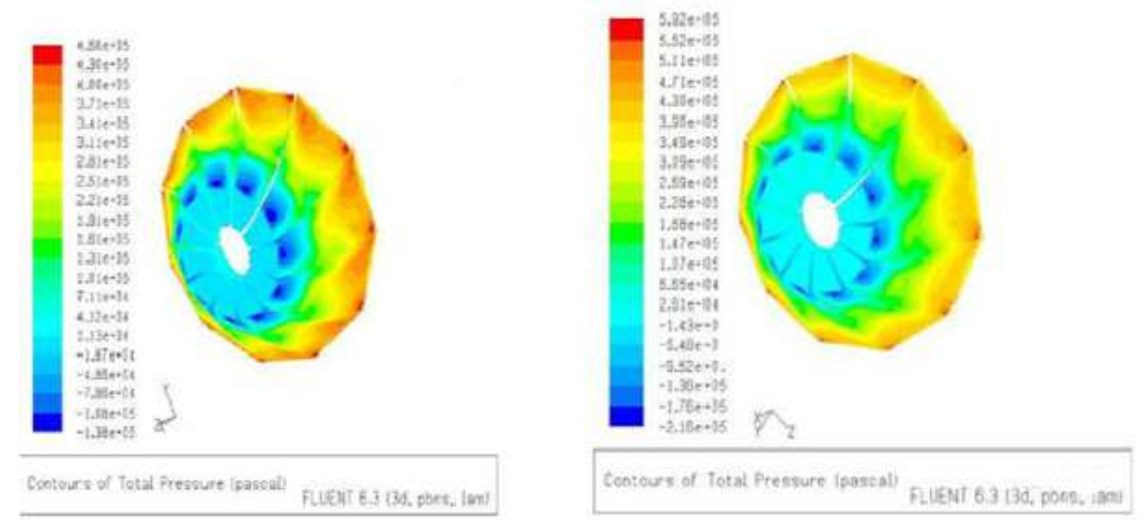

Figure 8: Total Pressure of Impeller without Slot Vs with Slot

From figure 7 and 8 the static pressure and the total pressure value of the impeller without and with slots shows that a better increase in the pressure at the tip of the impeller so that at the exit section of the compressor it might reach close to the required pressure ratio. 


\section{RESULTS AND DISCUSSIONS}

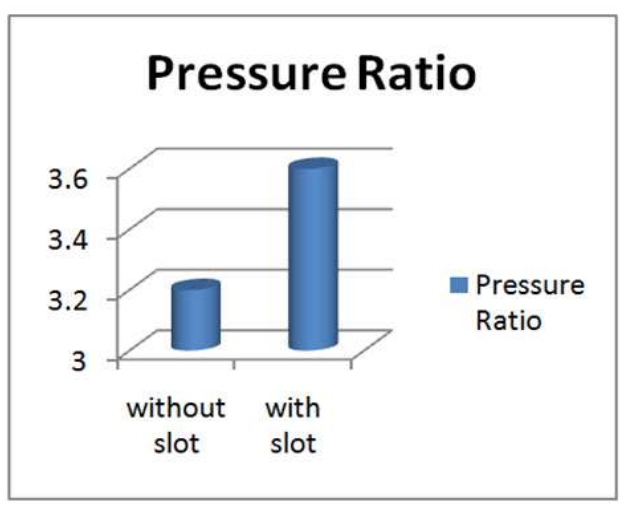

Figure 9: Pressure Ratio Comparison

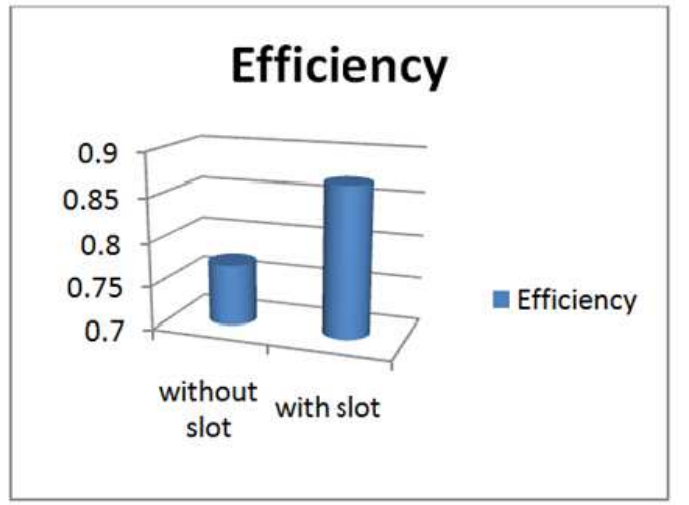

Figure 10: Efficiency Comparison

The analysed results gave great increase to pressure rise as well as the efficiency of the compressor. In future it is essential to get further improvement is sizing the dimension of slots and identifying the accurate place of flow slipping for better result.

\section{CONCLUSIONS}

The application of slots at a velocity falling point due to side slip controls the greater level of escaping fluid from its desired path. Hence at the tip of the impeller $90 \%$ of the kinetic energy have a possible to catch and convert into pressure energy to attain greater pressure level close to the requirement.

\section{REFERENCES}

1. G. Eason, B. Noble, and I.N. Sneddon, "On certain integrals of Lipschitz-Hankel type involving products of Bessel functions" Phil. Trans. Roy. Soc. London, vol. A247, pp. 529-551, April 1955. (references)

2. J. Clerk Maxwell, A Treatise on Electricity and Magnetism, 3rd ed., vol. 2. Oxford: Clarendon, 1892, pp.68-73.

3. I.S. Jacobs and C.P. Bean, "Fine particles, thin films and exchange anisotropy," in Magnetism, vol. III, G.T. Rado and H. Suhl, Eds. New York: Academic, 1963, pp. 271-350.

4. K. Elissa, "Title of paper if known," unpublished.

5. R. Nicole, "Title of paper with only first word capitalized," J. Name Stand. Abbrev., in press.

6. Sathish, T., "Experimental investigation on degradation of heat transfer properties of a black chromium-coated aluminium surface solar collector tube", International Journal of Ambient Energy, vol. 1, no. 1, pp. 1-5, 2018

7. Y. Yorozu, M. Hirano, K. Oka, and Y. Tagawa, "Electron spectroscopy studies on magneto-optical media and plastic substrate interface,” IEEE Transl. J. Magn. Japan, vol. 2, pp. 740-741, August 1987 [Digests 9th Annual Conf. Magnetics Japan, p. 301, 1982].

8. M. Young, The Technical Writer's Handbook. Mill Valley, CA: University Science, 1989. 